\title{
On a Hybrid Method for Generalized Mixed Equilibrium Problem and Fixed Point Problem of a Family of Quasi- $\phi$-Asymptotically Nonexpansive Mappings in Banach Spaces
}

\author{
Min Liu, Shih-sen Chang, and Ping Zuo \\ Department of Mathematics, Yibin University, Yibin, Sichuan 644007, China \\ Correspondence should be addressed to Shih-sen Chang, changss@yahoo.cn \\ Received 30 November 2009; Accepted 19 January 2010 \\ Academic Editor: Nanjing Huang
}

Copyright (C) 2010 Min Liu et al. This is an open access article distributed under the Creative Commons Attribution License, which permits unrestricted use, distribution, and reproduction in any medium, provided the original work is properly cited.

We prove a strong convergence theorem by using a hybrid method for finding a common element of the set of solutions for generalized mixed equilibrium problems, the set of fixed points of a family of quasi- $\phi$-asymptotically nonexpansive mappings in strictly convex reflexive Banach space with the Kadec-Klee property and, a Fréchet differentiable norm under weaker conditions. The method of the proof is different from, S. Takahashi and W. Takahashi that by (2008) and that by Takahashi and Zembayashi (2008) and see references. It also shows that the type of projection used in the iterative method is independent of the properties of the mappings. The results presented in the paper improve and extend some recent results.

\section{Introduction}

Let $E$ be a Banach space and let $C$ be a closed convex subsets of $E$. Let $F$ be an equilibrium bifunction from $C \times C$ into $R$, let $\psi: C \rightarrow R$ be a real-valued function, and let $A: C \rightarrow E^{*}$ be a nonlinear mapping. The "so-called" generalized mixed equilibrium problem is to find $z \in C$ such that

$$
F(z, y)+\langle A z, y-z\rangle+\psi(y)-\psi(z) \geq 0, \quad \forall y \in C, \mathrm{~s}
$$

The set of solutions of (1.1) is denoted by GMEP, that is,

$$
\mathrm{GMEP}=\{z \in C: F(z, y)+\langle A z, y-z\rangle+\psi(y)-\psi(z) \geq 0, \forall y \in C\}
$$




\section{Sepecial Examples}

(i) If $A=0$, then the problem (1.1) is equivalent to find $z \in C$ such that

$$
F(z, y)+\psi(y)-\psi(z) \geq 0, \quad \forall y \in C
$$

which is called the mixed equilibrium problem; see [1]. The set of solutions of (1.3) is denoted by MEP.

(ii) If $F=0$, then the problem (1.1) is equivalent to find $z \in C$ such that

$$
\langle A z, y-z\rangle+\psi(y)-\psi(z) \geq 0, \quad \forall y \in C,
$$

which is called the mixed variational inequality of Browder type. The set of solutions of (1.4) is denoted by $\operatorname{VI}(C, A, \psi)$.

(iii) If $\psi=0$, then the problem (1.1) is equivalent to find $z \in C$ such that

$$
F(z, y)+\langle A z, y-z\rangle \geq 0, \quad \forall y \in C
$$

which is called the generalized equilibrium problem; see [2]. The set of solutions of (1.5) is denoted by EP.

(iv) If $A=0, \psi=0$, then the problem (1.1) is equivalent to find $z \in C$ such that

$$
F(z, y) \geq 0, \quad \forall y \in C,
$$

which is called the equilibrium problem. The set of solutions of (1.6) is denoted by $\mathrm{EP}(\mathrm{F})$.

Recently, Tada and Takahashi [3] and S. Takahashi and W. Takahashi [4] considered iterative methods for finding an element of $\operatorname{EP}(F) \cap F(S)$ in Hilbert space. Very recently, S.Takahashi and W.Takahashi [2] introduced an iterative method for finding an element of $\mathrm{EP} \cap F(S)$, where $A: C \rightarrow H$ is an inverse-strongly monotone mapping and $S$ is nonexpansive mapping and then proved a strong convergence theorem in Hilbert space. On the other hand, Takahashi and Zembayashi [5] prove a strong convergence theorem for finding a common element of the set of solutions of an equilibrium problem and the set of fixed points of a relatively nonexpansive mapping in a Banach space by using the shrinking Projection method. Very recently, Kimura and Takahashi [6] prove a strong convergence theorem for a family of relatively nonexpansive mapping in a Banach space by using a hybrid method.

In this paper, motivated by Kimura and Takahashi [6], we prove a strong convergence theorem for finding an element of GMEP $\cap \bigcap_{\lambda \in \Lambda} F\left(T_{\lambda}\right)$ in Banach space by using a hybrid method, where $A: C \rightarrow E^{*}$ is a continuous and monotone operator and $T_{\lambda}(\lambda \in \Lambda)$ is a family of quasi- $\phi$-asymptotically nonexpansive mapping. Moreover, the method of proof adopted in the paper is different from that of $[2,5]$. 


\section{Preliminaries}

Throughout this paper, we assume that all the Banach spaces are real. We denote by $\mathbb{N}$ and $\mathbb{R}$ the sets of positive integers and real numbers, respectively. Let $E$ be a Banach space and let $E^{*}$ be the topological dual of $E$. For all $x \in E$ and $x^{*} \in E^{*}$, we denote the value of $x^{*}$ at $x$ by $\left\langle x, x^{*}\right\rangle$. The duality mapping $J: E \rightarrow 2^{E^{*}}$ is defined by

$$
J(x)=\left\{x^{*} \in E^{*}:\left\langle x, x^{*}\right\rangle=\|x\|^{2}=\left\|x^{*}\right\|^{2}\right\}, \quad x \in E .
$$

By Hahn-Banach theorem, $J(x)$ is nonempty; see [7] for more details. We denote the weak convergence and the strong convergence of a sequence $\left\{x_{n}\right\}$ to $x$ in $E$ by $x_{n} \rightarrow x$ and $x_{n} \rightarrow x$, respectively. A Banach space $E$ is said to be strictly convex if $\|x+y\| / 2<1$ for $x, y \in S(E)=$ $\{z \in E:\|z\|=1\}$ and $x \neq y$. It is also said to be uniformly convex if for each $\varepsilon \in(0,2]$ there exists $\delta>0$ such that $\|x+y\| / 2<1-\delta$ for $x, y \in S(E)$ and $\|x-y\| \geq \varepsilon$. E is said to have the Kadec-Klee property, that is, for any sequence $\left\{x_{n}\right\} \subset E$, if $x_{n} \rightarrow x \in E$ and $\left\|x_{n}\right\| \rightarrow\|x\|$, then $x_{n} \rightarrow x$.

Define $f: S(E) \times S(E) \times \mathbb{R} \backslash\{0\} \rightarrow \mathbb{R}$ by

$$
f(x, y, t)=\frac{\|x+t y\|-\|x\|}{t}
$$

for $x, y \in S(E)$ and $t \in \mathbb{R} \backslash\{0\}$. A norm of $E$ is said to be Gâteaux differentiable if $\lim _{t \rightarrow 0} f(x, y, t)$ has a limit for each $x, y \in S(E)$. In this case, $E$ is said to be smooth. A norm of $E$ is said to be Fréchet differentiable if $\lim _{t \rightarrow 0} f(x, y, t)$ is attained uniformly for $y \in S(E)$ for each $x \in S(E)$. It is known that $E^{*}$ has a Fréchet differentiable norm if and only if $E$ is strictly convex and reflexive, and has the Kadec-Klee property. We know that if $E$ is smooth, strictly convex, and reflexive, then the duality mapping $J$ is single valued, one to one, and onto. In this case, the inverse mapping $J^{-1}$ coincides with the duality mapping $J^{*}$ on $E^{*}$. See [8] for more details.

Remark 2.1. If $E$ is a reflexive and strictly convex Banach space, then $J^{-1}$ is hemicontinuous, that is, $J^{-1}$ is norm-weak continuous.

Let $E$ be a smooth, strictly convex and reflexive Banach space and let $C$ be a closed convex subset of $E$. Throughout this paper, we denote by $\phi$ the function defined by

$$
\phi(y, x)=\|y\|^{2}-2\langle y, J x\rangle+\|x\|^{2}, \quad \forall x, y \in E
$$

Let $\left\{C_{n}\right\}$ be a sequence of nonempty closed convex subset of a reflexive Banach space $E$. We define two subsets $s-L i_{n} C_{n}$ and $w-L s_{n} C_{n}$ as follows: $x \in s-L i_{n} C_{n}$ if and only if there exists $\left\{x_{n}\right\} \subset E$ such that $\left\{x_{n}\right\}$ converges strongly to $x$ and that $x_{n} \in C_{n}$ for all $n \in \mathbb{N}$. Similarly, $y \in w-L s_{n} C_{n}$ if and only if there exists a subsequence $\left\{C_{n_{i}}\right\}$ of $\left\{C_{n}\right\}$ and a sequence $\left\{y_{i}\right\} \subset E$ such that $\left\{y_{i}\right\}$ converges weakly to $y$ and $y_{i} \in C_{n_{i}}$ for all $i \in \mathbb{N}$. We define the Mosco convergence [9] of $\left\{C_{n}\right\}$ as follows: if $C_{0}$ satisfies that $C_{0}=s-L i_{n} C_{n}=w-L s_{n} C_{n}$, then it is said that $\left\{C_{n}\right\}$ converges to $C_{0}$ in the sense of Mosco and we write $C_{0}=M-\lim _{n \rightarrow \infty} C_{n}$. For more details, see [10]. 
Let $C$ be a nonempty closed convex subset of a smooth, strictly convex and reflexive Banach space $E$. Then, for arbitrarily fixed $x \in E$, a function $C \ni y \mapsto\|x-y\|^{2} \in \mathbb{R}$ has a unique minimizer $y_{x} \in C$. Using such a point, we define the metric projection $P_{C}$ by $P_{C} x=$ $y_{x}=\arg \min _{y \in C}\|x-y\|^{2}$ for every $x \in E$. In a similar fashion, we can see that a function $C \ni y \mapsto \phi(x, y) \in \mathbb{R}$ has a unique minimizer $z_{x} \in C$. The generalized projection $\Pi_{C}$ of $E$ onto $C$ is defined by $\Pi_{C}=z_{x}=\arg \min _{y \in C} \phi(x, y)$ for every $x \in E$; see [11]. satisfies

The generalized projection $\Pi_{C}$ from $E$ onto $C$ is well defined and single valued and

$$
(\|x\|-\|y\|)^{2} \leq \phi(y, x) \leq(\|x\|+\|y\|)^{2}, \quad \forall x, y \in E .
$$

If $E$ is a Hilbert space, then $\phi(y, x)=\|y-x\|^{2}$ and $\Pi_{C}$ is the metric projection $P_{C}$ of $E$ onto $C$. It is well-known that the following conclusions hold.

Lemma 2.2 (see[11, 12]). Let $C$ be a nonempty closed convex subsets of a smooth, strictly convex and reflexive Banach spaces. Then

$$
\phi\left(x, \Pi_{C} y\right)+\phi\left(\Pi_{C} y, y\right) \leq \phi(x, y), \quad \forall x \in C, y \in E
$$

Lemma 2.3. Let $C$ be a nonempty closed convex subsets of a smooth, strictly convex and reflexive Banach spaces $E$, let $x \in E$ and let $z \in C$. Then the following conclusions hold:

(a) $z=\Pi_{C} x \Leftrightarrow\langle y-z, J x-J z\rangle \leq 0$, for all $y \in C$.

(b) For $x, y \in E, \phi(x, y)=0$ if and only if $x=y$.

The following theorem proved by Tsukada [13] plays an important role in our results.

Theorem 2.4. Let $E$ be a smooth, reflexive, and strictly convex Banach space having the Kadec-Klee property. Let $\left\{K_{n}\right\}$ be a sequence of nonempty closed convex subset of $E$. If $K_{0}=M-\lim _{n \rightarrow \infty} K_{n}$ exists and is nonempty, then $\left\{P_{K_{n}} x\right\}$ converges strongly to $P_{K_{0}} x$ for each $x \in C$.

Theorem 2.4 is still valid if we replace the metric projections with the generalized projections as follows:

Theorem 2.5. Let E be a smooth, reflexive, and strictly convex Banach spaces having the Kadec-Klee property. Let $\left\{K_{n}\right\}$ be a sequence of nonempty closed convex subsets of $E$. If $K_{0}=M-\lim _{n \rightarrow \infty} K_{n}$ exists and is nonempty, then $\left\{\Pi_{K_{n}} x\right\}$ converges strongly to $\Pi_{K_{0}} x$ for each $x \in C$.

Let $C$ be a nonempty closed convex subsets of $E$, and let $T$ be a mapping from $C$ into itself. We denoted by $F(T)$ the set of fixed points of $T$. $T$ is said to be $\phi$-asymptotically nonexpansive, if there exists some real sequence $\left\{k_{n}\right\}$ with $k_{n} \geq 1$ and $k_{n} \rightarrow 1$ such that $\phi\left(T^{n} x, T^{n} y\right) \leq k_{n} \phi(x, y)$ for all $n \geq 1$ and $x, y \in C$. $T$ is said to be quasi- $\phi$-asymptotically nonexpansive [14], if there exists some real sequence $\left\{k_{n}\right\}$ with $k_{n} \geq 1$ and $k_{n} \rightarrow 1$ and $F(T) \neq \emptyset$ such that $\phi\left(p, T^{n} x\right) \leq k_{n} \phi(p, x)$ for all $n \geq 1, x \in C$, and $p \in F(T)$. $T$ is said to be uniformly Lipschitzian continuous if there exists some $L>0$ such that $\left\|T^{n} x-T^{n} y\right\| \leq L\|x-y\|$ for all $n \geq 1$ and $x, y \in C$. A point $p \in C$ is said to be an asymptotic fixed point of $T[15,16]$ if there exists $\left\{x_{n}\right\}$ in $C$ which converges weakly to $p$ and $\lim _{n \rightarrow \infty}\left\|x_{n}-T x_{n}\right\|=0$. We denote the set of all asymptotic fixed point of $T$ by $\widehat{F}(T)$. Following Matsushita and Takahashi [17], 
a mapping $T: C \rightarrow C$ is said to be relatively nonexpansive if the following conditions are satisfied:

(1) $F(T)$ is nonempty,

(2) $\phi(u, T x) \leq \phi(u, x)$, for all $u \in F(T), x \in C$,

(3) $\widehat{F}(T)=F(T)$.

A mapping $T: C \rightarrow C$ is said to be quasi- $\phi$-nonexpansive, if $\phi(p, T x) \leq$ $\phi(p, x)$, for all $x \in C$, for all $p \in F(T)$.

We remark that a quasi- $\phi$-nonexpansive mapping with a nonempty fixed point set $F(T)$ is a quasi- $\phi$-asymptotically nonexpansive mapping, but the converse may be not true.

A mapping $T: C \rightarrow C$ is said to be closed, if for any sequence $\left\{x_{n}\right\} \subset C$ with $x_{n} \rightarrow x$ and $T x_{n} \rightarrow y, T x=y$.

Lemma 2.6. Let E be a strictly convex reflexive Banach space having the Kadec-Klee property and a Fréchet differentiable norm, $C$ be a nonempty closed convex subset of $E$, and let $T$ be a uniformly Lipschitzian continuous and quasi- $\phi$-asymptotically nonexpansive mapping from $C$ into itself. Then $F(T)$ is closed and convex.

Proof. We first show that $F(T)$ is closed. To see this, let $\left\{p_{n}\right\}$ be a sequence in $F(T)$ with $p_{n} \rightarrow p$ as $n \rightarrow \infty$; we shall prove that $p \in F(T)$. In fact, from the definition of $T$, we have $\phi\left(p_{n}, T p\right) \leq k_{1} \phi\left(p_{n}, p\right) \rightarrow 0(n \rightarrow \infty)$. Therefore we have

$$
\begin{aligned}
\lim _{n \rightarrow \infty} \phi\left(p_{n}, T p\right) & =\lim _{n \rightarrow \infty}\left(\left\|p_{n}\right\|^{2}-2\left\langle p_{n}, J T p\right\rangle+\|T p\|^{2}\right) \\
& =\|p\|^{2}-2\langle p, J T p\rangle+\|T p\|^{2}=\phi(p, T p)=0,
\end{aligned}
$$

that is, $p=T p$. We next show that $F(T)$ is convex. To end this, for arbitrary $p, q \in F(T), t \in$ $(0,1)$, by setting $w=t p+(1-t) q$, it is sufficient to show that $T w=w$. Indeed, by using (2.3) we have

$$
\begin{aligned}
\phi\left(w, T^{n} w\right) & =\|w\|^{2}-2\left\langle w, J T^{n} w\right\rangle+\left\|T^{n} w\right\|^{2} \\
& =\|w\|^{2}-2 t\left\langle p, J T^{n} w\right\rangle-2(1-t)\left\langle q, J T^{n} w\right\rangle+\left\|T^{n} w\right\|^{2} \\
& =\|w\|^{2}+t \phi\left(p, T^{n} w\right\rangle+(1-t) \phi\left(q, T^{n} w\right\rangle-t\|p\|^{2}-(1-t)\|q\|^{2} \\
& \leq\|w\|^{2}+k_{n} t \phi(p, w)+k_{n}(1-t) \phi(q, w)-t\|p\|^{2}-(1-t)\|q\|^{2} \\
& =\left(k_{n}-1\right)\left(t\|p\|^{2}+(1-t)\|q\|^{2}-\|w\|^{2}\right),
\end{aligned}
$$

which implies that $\phi\left(w, T^{n} w\right) \rightarrow 0$ as $n \rightarrow \infty$. From (2.4) we have $\left\|T^{n} w\right\| \rightarrow\|w\|$. Consequently $\left\|J T^{n} w\right\| \rightarrow\|J w\|$. This implies that $\left\{J T^{n} w\right\}$ is bounded in $E^{*}$. Since $E$ is reflexive, so is $E^{*}$, we can assume that

$$
J T^{n} w \rightarrow f_{0} \in E^{*}
$$


In view of the reflexive of $E$, we see that $J(E)=E^{*}$. Hence there exists $p \in E$ such that $J p=f_{0}$. By virtue of the weak lower semicontinuity of norm $\|\cdot\|$, we have

$$
\begin{aligned}
0=\liminf _{n \rightarrow \infty} \phi\left(w, T^{n} w\right) & =\liminf _{n \rightarrow \infty}\left(\|w\|^{2}-2\left\langle w, J\left(T^{n} w\right)\right\rangle+\left\|T^{n} w\right\|^{2}\right) \\
& =\liminf _{n \rightarrow \infty}\left(\|w\|^{2}-2\left\langle w, J\left(T^{n} w\right)\right\rangle+\left\|J\left(T^{n} w\right)\right\|^{2}\right) \\
& \geq\|w\|^{2}-2\left\langle w, f_{0}\right\rangle+\left\|f_{0}\right\|^{2} \\
& =\|w\|^{2}-2\langle w, J p\rangle+\|J p\|^{2} \\
& =\|w\|^{2}-2\langle w, J p\rangle+\|p\|^{2}=\phi(w, p),
\end{aligned}
$$

that is, $w=p$. This implies that $f_{0}=J w$. Thus from (2.8) we have $J T^{n} w \rightarrow J w \in E^{*}$. Since $\left\|J T^{n} w\right\| \rightarrow\|J w\|$ and $E^{*}$ has the Kadec-Klee property, we have $J T^{n} w \rightarrow J w$. Note that $J^{-1}: E^{*} \rightarrow E$ is hemicontinuous, it yields that $T^{n} w \rightarrow w$. Again since $\left\|T^{n} w\right\| \rightarrow\|w\|$, by using the Kadec-Klee property of $E$, we have $T^{n} w \rightarrow w$. Hence $T T^{n} w=T^{n+1} w \rightarrow w$ as $n \rightarrow \infty$. Since $T$ is uniformly Lipschitzian continuous, we have $w=T w$. This completes the proof.

For solving the equilibrium problem for bifunction $F: C \times C \rightarrow \mathbb{R}$, let us assume that $F$ satisfies the following conditions:

$\left(A_{1}\right) F(x, x)=0$ for all $x \in C$,

$\left(A_{2}\right) F$ is monotone, that is, $F(x, y)+F(y, x) \leq 0$ for all $x, y \in C$,

$\left(A_{3}\right)$ for each $x, y, z \in C$,

$$
\limsup _{t \downarrow 0} F(t z+(1-t) x, y) \leq F(x, y)
$$

$\left(A_{4}\right)$ for each $x \in C, y \mapsto F(x, y)$ is a convex and lower semicontinuous.

If an equilibrium bifunction $F: C \times C \rightarrow R$ satisfies conditions $\left(A_{1}\right)-\left(A_{4}\right)$, then we have the following two important results.

Lemma 2.7 (see[18]). Let $C$ be a nonempty closed convex subset of a smooth, strictly convex and reflexive Banach space $E$, let $F$ be an equilibrium bifunction from $C \times C$ to $\mathbb{R}$ satisfying conditions $\left(A_{1}\right)-\left(A_{4}\right)$, let $r>0$, and let $x \in E$. Then, there exists $z \in C$ such that

$$
F(z, y)+\frac{1}{r}\langle y-z, J z-J x\rangle \geq 0, \quad \forall y \in C
$$

Lemma 2.8 (see[5]). Let $C$ be a nonempty closed convex subset of a uniformly smooth, strictly convex and reflexive Banach space $E$, and let $F: C \times C \rightarrow \mathbb{R}$ be an equilibrium bifunction satisfying conditions $\left(A_{1}\right)-\left(A_{4}\right)$. For $r>0$ and $x \in E$, define a mapping $T_{r}: E \rightarrow C$ as follows:

$$
T_{r}(x)=\left\{z \in C: F(z, y)+\frac{1}{r}\langle y-z, J z-J x\rangle \geq 0, \forall y \in C\right\},
$$


for all $x \in E$. Then, the following hold:

(1) $T_{r}$ is single-valued,

(2) $T_{r}$ is a firmly nonexpansive-type mapping, that is, for any $x, y \in E$,

$$
\left\langle T_{r} x-T_{r} y, J T_{r} x-J T_{r} y\right\rangle \leq\left\langle T_{r} x-T_{r} y, J x-J y\right\rangle,
$$

(3) $F\left(T_{r}\right)=\widehat{F}\left(T_{r}\right)=\operatorname{EP}(F)$,

(4) $\mathrm{EP}(F)$ is a closed and convex set.

Lemma 2.9 (see[5]). Let $C$ be a nonempty closed convex subset of a smooth, strictly convex and reflexive Banach space $E$, and let $F: C \times C \rightarrow \mathbb{R}$ be an equilibrium bifunction satisfying conditions $\left(A_{1}\right)-\left(A_{4}\right)$. For $r>0, x \in E$ and $q \in F\left(T_{r}\right)$,

$$
\phi\left(q, T_{r} x\right)+\phi\left(T_{r} x, x\right) \leq \phi(q, x) .
$$

\section{The Main Results}

Lemma 3.1. Let E be a strictly convex reflexive Banach space having a Fréchet differentiable norm, $C$ a nonempty closed convex subset of $E$, and $\left\{S_{n}\right\}$ a sequence of mappings of $C$ into itself. Let $\left\{x_{n}\right\}$ be a strongly convergent sequence in $C$ with a limit $x_{0}$ and $\left\{y_{n}\right\}$ a sequence in $C$ defined by $y_{n}=$ $J^{*}\left(\alpha_{n} J x_{n}+\left(1-\alpha_{n}\right) J S_{n} x_{n}\right)$ for each $n \in \mathbb{N}$, where $\left\{\alpha_{n}\right\}$ is a convergent sequence in $[0,1]$ with a limit $\alpha_{0} \in[0,1)$. Suppose that $\phi\left(x_{0}, y_{n}\right) \leq \phi\left(x_{0}, x_{n}\right)+\xi_{n}$ for all $n \in \mathbb{N}$ and that $\left\{J y_{n}\right\}$ converges weakly to $y_{0}^{*} \in E^{*}$, where $\lim _{n \rightarrow \infty} \xi_{n}=0$. Then $\left\{J x_{n}-J S_{n} x_{n}\right\}$ converges strongly to 0 . Moreover, if $E$ has the Kadec-Klee property, then $\left\{S_{n} x_{n}\right\}$ converges strongly to $x_{0}$.

Proof. Since $\phi\left(x_{0}, y_{n}\right) \leq \phi\left(x_{0}, x_{n}\right)+\xi_{n}$ for $n \in \mathbb{N}$, we have that

$$
0 \leq \lim _{n \rightarrow \infty} \phi\left(x_{0}, y_{n}\right) \leq \lim _{n \rightarrow \infty} \phi\left(x_{0}, x_{n}\right)+\xi_{n}=0 .
$$

and hence $\lim _{n \rightarrow \infty} \phi\left(x_{0}, y_{n}\right)=0$. Since

$$
\left(\left\|x_{0}\right\|-\left\|y_{n}\right\|\right)^{2}=\left\|x_{0}\right\|^{2}-2\left\|x_{0}\right\|\left\|y_{n}\right\|+\left\|y_{n}\right\|^{2} \leq \phi\left(x_{0}, y_{n}\right)
$$

for $n \in \mathbb{N}$, we have that $\lim _{n \rightarrow \infty}\left\|y_{n}\right\|=\left\|x_{0}\right\|$ and that

$$
\lim _{n \rightarrow \infty}\left\langle x_{0}, J y_{n}\right\rangle=\lim _{n \rightarrow \infty} \frac{1}{2}\left(\left\|x_{0}\right\|^{2}+\left\|y_{n}\right\|^{2}-\phi\left(x_{0}, y_{n}\right)\right)=\left\|x_{0}\right\|^{2}
$$

Using weak lower semicontinuity of the norm, we have that

$$
\begin{aligned}
\left\|x_{0}\right\|^{2} & =\lim _{n \rightarrow \infty}\left\langle x_{0}, J y_{n}\right\rangle=\left\langle x_{0}, y_{0}^{*}\right\rangle \leq\left\|x_{0}\right\|\left\|y_{0}^{*}\right\| \leq\left\|x_{0}\right\| \liminf _{n \rightarrow \infty}\left\|J y_{n}\right\| \\
& =\left\|x_{0}\right\| \lim _{n \rightarrow \infty}\left\|J y_{n}\right\|=\left\|x_{0}\right\|^{2} .
\end{aligned}
$$


Therefore, we have that $\left\|y_{0}^{*}\right\|^{2}=\left\langle x_{0}, y_{0}^{*}\right\rangle=\left\|x_{0}\right\|^{2}$, and hence $y_{0}^{*}=J x_{0}$. Thus we have that $\left\{J y_{n}\right\}$ converges weakly to $J x_{0}$. It also holds that

$$
\lim _{n \rightarrow \infty}\left\|J y_{n}\right\|=\lim _{n \rightarrow \infty}\left\|y_{n}\right\|=\left\|x_{0}\right\|=\left\|J x_{0}\right\|
$$

Since $E$ has a Fréchet differentiable norm, it follows that $E^{*}$ has the Kadec-Klee property, and thus we have that $\left\{J y_{n}\right\}$ converges strongly to $J x_{0}$. Then, we have that

$$
\begin{aligned}
\left\|J x_{0}-J y_{n}\right\| & =\left\|J x_{0}-\left(\alpha_{n} J x_{n}+\left(1-\alpha_{n}\right) J S_{n} x_{n}\right)\right\| \\
& \geq\left\|J x_{0}-\alpha_{n} J x_{0}-\left(1-\alpha_{n}\right) J S_{n} x_{n}\right\|-\alpha_{n}\left\|J x_{n}-J x_{0}\right\| \\
& =\left(1-\alpha_{n}\right)\left\|J x_{0}-J S_{n} x_{n}\right\|-\alpha_{n}\left\|J x_{n}-J x_{0}\right\|
\end{aligned}
$$

for $n \in \mathbb{N}$. Using norm-to-norm continuity of $J$, we get that

$$
\lim _{n \rightarrow \infty}\left(1-\alpha_{n}\right)\left\|J x_{0}-J S_{n} x_{n}\right\|=\left(1-\alpha_{0}\right) \lim _{n \rightarrow \infty}\left\|J x_{0}-J S_{n} x_{n}\right\|=0,
$$

and since $\alpha_{0}<1$, we have that

$$
\lim _{n \rightarrow \infty}\left\|J x_{0}-J S_{n} x_{n}\right\|=0
$$

We also have that $\left\{J S_{n} x_{n}\right\}$ converges strongly to $J x_{0}$, and hence we obtain that $\left\{J x_{n}-J S_{n} x_{n}\right\}$ converges strongly to 0 . Further, let us suppose that $E$ has the Kadec-Klee property. Then, the norm of $E^{*}$ is Fréchet differentiable and, therefore, $J^{*}$ is norm-to-norm continuous. Hence we have that

$$
\lim _{n \rightarrow \infty}\left\|x_{0}-S_{n} x_{n}\right\|=\lim _{n \rightarrow \infty}\left\|J^{*} J x_{0}-J^{*} J S_{n} x_{n}\right\|=0,
$$

which is the desired result.

Theorem 3.2. Let $E$ be a strictly convex reflexive Banach space having the Kadec-Klee property and a Fréchet differentiable norm, $C$ a nonempty closed convex subset of $E, A: C \rightarrow E^{*}$ a continuous and monotone mapping, $\psi: C \rightarrow \mathbb{R}$ a lower semicontinuous and convex function $F$ a bifunction from $C \times C$ to $\mathbb{R}$ which satisfies the conditions $\left(A_{1}\right)-\left(A_{4}\right)$, and $\left\{T_{\lambda}\right\}(\lambda \in \Lambda): C \rightarrow C$ a family of uniformly Lipschitzian continuous and quasi- $\phi$-asymptotically nonexpansive mappings such that 
$F=\bigcap_{\lambda \in \Lambda} F\left(T_{\lambda}\right) \cap G M E P \neq \emptyset$. Assume that $R=\sup \{\|u\|: u \in F\}<\infty$. Let $\left\{x_{n}\right\}$ be the sequence generated by $x_{1}=x \in C, C_{1}=C$, and

$$
\begin{gathered}
y_{n}(\lambda)=J^{*}\left(\alpha_{n} J x_{n}+\left(1-\alpha_{n}\right) J T_{\lambda}^{n} x_{n}\right) \quad \forall \lambda \in \Lambda, \\
u_{n}(\lambda) \in C \text { such that } \\
F\left(u_{n}(\lambda), y\right)+\left\langle A u_{n}(\lambda), y-u_{n}(\lambda)\right\rangle+\psi(y)-\psi\left(u_{n}(\lambda)\right)+\frac{1}{r_{n_{\lambda}}}\left\langle y-u_{n}(\lambda), J u_{n}(\lambda)-J y_{n}(\lambda)\right\rangle \geq 0, \\
C_{n+1}=\{z \in C, \lambda \in \Lambda \\
\left.z \in C_{n}: \sup _{\lambda \in \Lambda} \phi\left(z, u_{n}(\lambda)\right) \leq \phi\left(z, x_{n}\right)+\xi_{n}\right\}, \\
x_{n+1}=P_{C_{n+1}} x, \quad \forall n \geq 0,
\end{gathered}
$$

where $J$ is the duality mapping on $E$ and $\xi_{n}=\left(1-\alpha_{n}\right)\left(\sup _{\lambda \in \Lambda} k_{n}(\lambda)-1\right) \sup _{u \in F} \phi\left(u, x_{n}\right)$ for all $x_{n} \in$ $C$, where $\lim _{n \rightarrow \infty} \sup _{\lambda \in \Lambda} k_{n}(\lambda)=1$. Let $\left\{\alpha_{n}\right\}$ be a sequence in $[0,1]$ such that $\lim _{\inf } \operatorname{si\infty }_{n \rightarrow \infty} \alpha_{n}<1$ and $\left\{r_{n_{\lambda}}\right\} \subset[a, \infty)$ for some $a>0$, then $\left\{x_{n}\right\}$ converge strongly to $P_{F} x$, where $P_{F}$ is the metric projection of $E$ onto $F$.

Proof. We define a bifunction $G: C \times C \rightarrow R$ by

$$
G(z, y)=F(z, y)+\langle A z, y-z\rangle+\psi(y)-\psi(z), \quad \forall z, y \in C
$$

Next, we prove that the bifunction $G$ satisfies conditions $\left(A_{1}\right)-\left(A_{4}\right)$ as follows

$\left(A_{1}\right) G(x, x)=0$ for all $x \in C$, since $G(x, x)=F(x, x)+\langle A x, 0\rangle+\psi(x)-\psi(x)=F(x, x)=0$, for all $x \in C$.

$\left(A_{2}\right) G$ is monotone, that is, $G(z, y)+G(y, z) \leq 0$ for all $y, z \in C$.

Since $A$ is a continuous and monotone operator, hence from the definition of $G$ we have

$$
\begin{aligned}
G(z, y)+G(y, z)= & F(z, y)+\langle A z, y-z\rangle+\psi(y)-\psi(z)+F(y, z) \\
& +\langle A y, z-y\rangle+\psi(z)-\psi(y) \\
= & F(z, y)+F(y, z)+\langle A z, y-z\rangle-\langle A y, y-z\rangle \\
\leq & 0+\langle A z-A y, y-z\rangle \\
= & -\langle A y-A z, y-z\rangle \\
\leq & 0 .
\end{aligned}
$$


$\left(A_{3}\right)$ for each $x, y, z \in C$,

$$
\limsup _{t ! 0} G(t z+(1-t) x, y) \leq G(x, y)
$$

Since $A$ is continuous and $\psi$ is lower semicontinuous, we have

$$
\begin{aligned}
& \underset{t \downarrow 0}{\limsup } G(t z+(1-t) x, y) \\
& =\limsup _{t \downarrow 0} F(t z+(1-t) x, y)+\limsup _{t \downarrow 0}\langle A(t z+(1-t) x), y-(t z+(1-t) x)\rangle \\
& \quad+\limsup _{t \downarrow 0}[\psi(y)-\psi(t z+(1-t) x)] \\
& \leq F(x, y)+\langle A x, y-x\rangle+\psi(y)-\psi(x)=G(x, y) .
\end{aligned}
$$

$\left(A_{4}\right)$ For each $x \in C, y \mapsto G(x, y)$ is a convex and lower semicontinuous.

For each $x \in C$, for all $t \in(0,1)$ and for all $y, z \in C$, since $F$ satisfies $\left(\mathrm{A}_{4}\right)$ and $\psi$ is convex, we have

$$
\begin{aligned}
& G(x, t y+(1-t) z)=F(x, t y+(1-t) z)+\langle A x, t y+(1-t) z-x\rangle+\psi(t y+(1-t) z)-\psi(x) \\
& \quad \leq t[F(x, y)+\langle A x, y-x\rangle+\psi(y)-\psi(x)]+(1-t)[F(x, z)+\langle A x, z-x\rangle+\psi(z)-\psi(x)] \\
& \quad=t G(x, y)+(1-t) G(x, z) .
\end{aligned}
$$

So, $y \mapsto G(x, y)$ is convex.

Similarly, we can prove that $y \mapsto G(x, y)$ is lower semi-continuous.

Therefore, the generalized mixed equilibrium problem (1.1) is equivalent to the following equilibrium problem: find $z \in C$ such that

$$
G(z, y) \geq 0, \quad \forall y \in C,
$$

then, GMEP $=\operatorname{EP}(G)$. We have $F=\operatorname{GMEP} \cap \bigcap_{\lambda \in \Lambda} F\left(T_{\lambda}\right)=\operatorname{EP}(G) \cap \bigcap_{\lambda \in \Lambda} F\left(T_{\lambda}\right)$. So, (3.10) can be written as

$$
y_{n}(\lambda)=J^{*}\left(\alpha_{n} J x_{n}+\left(1-\alpha_{n}\right) J T_{\lambda}^{n} x_{n}\right) \quad \forall \lambda \in \Lambda,
$$

$u_{n}(\lambda) \in C$ such that $G\left(u_{n}(\lambda), y\right)+\frac{1}{r_{n_{\lambda}}}\left\langle y-u_{n}(\lambda), J u_{n}(\lambda)-J y_{n}(\lambda)\right\rangle \geq 0, \quad \forall y \in C, \lambda \in \Lambda$,

$$
\begin{gathered}
C_{n+1}=\left\{z \in C_{n}: \sup _{\lambda \in \Lambda} \phi\left(z, u_{n}(\lambda)\right) \leq \phi\left(z, x_{n}\right)+\xi_{n}\right\}, \\
x_{n+1}=P_{C_{n+1}} x, \quad \forall n \geq 0 .
\end{gathered}
$$


Since the bifunction $G$ satisfies conditions $\left(A_{1}\right)-\left(A_{4}\right)$, from Lemma 2.8, for given $r>0$ and $x \in E$, we can define a mapping $W_{r}: E \rightarrow 2^{C}$ as follows:

$$
W_{r}(x)=\left\{z \in C: G(z, y)+\frac{1}{r}\langle y-z, J z-J x\rangle \geq 0, \forall y \in C\right\}
$$

Moreover, $W_{r}$ satisfies the conclusions in Lemma 2.8.

Putting $u_{n}(\lambda)=W_{r_{n}} y_{n}(\lambda)$ for all $n \in \mathbb{N}$, we have from Lemma 2.8 and Lemma 2.9 that $W_{r_{n_{\lambda}}}$ is relatively nonexpansive.

We divide the proof of Theorem 3.2 into five steps.

Step 1. We first show that $C_{n}$ is closed and convex for every $n \in \mathbb{N}$. From the definition of $\phi$, we may show that

$$
\begin{aligned}
C_{n+1} & =\left\{z \in C_{n}: \sup _{\lambda \in \Lambda} \phi\left(z, u_{n}(\lambda)\right) \leq \phi\left(z, x_{n}\right)+\xi_{n}\right\} \\
& =\bigcap_{\lambda \in \Lambda}\left\{z \in C_{n}: \phi\left(z, u_{n}(\lambda)\right) \leq \phi\left(z, x_{n}\right)+\xi_{n}\right\} \\
& =\bigcap_{\lambda \in \Lambda}\left\{z \in C: 2\left\langle z, J x_{n}-J u_{n}(\lambda)\right\rangle+\left\|u_{n}(\lambda)\right\|^{2}-\left\|x_{n}\right\|^{2}-\xi_{n} \leq 0\right\} \cap C_{n},
\end{aligned}
$$

and thus $C_{n}$ is closed and convex for every $n \in \mathbb{N}$.

Step 2. Next we show that $F \subset C_{n}$ for each $n \in \mathbb{N}$ and $\lambda \in \Lambda$.

for any $u \in F$, since $W_{r_{n_{\lambda}}}$ is relatively nonexpansive and $\left\{T_{\lambda}\right\}, \lambda \in \Lambda$ is quasi- $\phi$ asymptotically nonexpansive, we have

$$
\begin{aligned}
\phi\left(u, u_{n}(\lambda)\right) & =\phi\left(u, W_{r_{n_{\lambda}}} y_{n}(\lambda)\right) \\
& \leq \phi\left(u, y_{n}(\lambda)\right) \\
& =\phi\left(u, J^{*}\left(\alpha_{n} J x_{n}+\left(1-\alpha_{n}\right) J T_{\lambda}^{n} x_{n}\right)\right) \\
& =\|u\|^{2}-2\left\langle u, \alpha_{n} J x_{n}+\left(1-\alpha_{n}\right) J T_{\lambda}^{n} x_{n}\right\rangle+\left\|\alpha_{n} J x_{n}+\left(1-\alpha_{n}\right) J T_{\lambda}^{n} x_{n}\right\|^{2} \\
& \leq\|u\|^{2}-2 \alpha_{n}\left\langle u, J x_{n}\right\rangle-2\left(1-\alpha_{n}\right)\left\langle u, J T_{\lambda}^{n} x_{n}\right\rangle+\alpha_{n}\left\|J x_{n}\right\|^{2}+\left(1-\alpha_{n}\right)\left\|J T_{\lambda}^{n} x_{n}\right\|^{2} \\
& =\alpha_{n} \phi\left(u, x_{n}\right)+\left(1-\alpha_{n}\right) \phi\left(u, T_{\lambda}^{n} x_{n}\right) \\
& \leq \alpha_{n} \phi\left(u, x_{n}\right)+\left(1-\alpha_{n}\right) k_{n}(\lambda) \phi\left(u, x_{n}\right) \\
& =\phi\left(u, x_{n}\right)+\left(1-\alpha_{n}\right)\left(k_{n}(\lambda)-1\right) \phi\left(u, x_{n}\right) \\
& \leq \phi\left(u, x_{n}\right)+\xi_{n} .
\end{aligned}
$$


Hence, we have $\sup _{\lambda \in \Lambda} \phi\left(u, u_{n}(\lambda)\right) \leq \phi\left(u, x_{n}\right)+\xi_{n}$, that is, $u \in C_{n}$. This implies that

$$
F \subset C_{n}, \quad \forall n \in \mathbb{N}
$$

Step 3. Now we prove that the $\operatorname{limit}_{\lim _{n \rightarrow \infty}} x_{n}$ exists.Since $F$ is nonempty, $C_{n}$ is a nonempty closed convex subset of $E$, and, thus, $P_{C_{n}}$ exists for every $n \in \mathbb{N}$, hence $\left\{x_{n}\right\}$ is well defined. Also, since $\left\{C_{n}\right\}$ is a decreasing sequence of closed convex subsets of $E$ such that $C_{0}=\bigcap_{n=1}^{\infty} C_{n}$ is nonempty, it follows that

$$
M-\lim _{n \rightarrow \infty} C_{n}=C_{0}=\bigcap_{n=1}^{\infty} C_{n} \neq \emptyset .
$$

By Theorem 2.4, $\left\{x_{n}\right\}=\left\{P_{C_{n}} x\right\}$ converges strongly to $x_{0}=P_{C_{0}} x$. Therefore, we have

$$
\left\|x_{n+1}-x_{n}\right\| \longrightarrow 0
$$

Step 4. Next we prove that $x_{0} \in F$.

(a) First, we prove that $x_{0} \in \bigcap_{\lambda \in \Lambda} F\left(T_{\lambda}\right)$.

Since $x_{0} \in C_{n}$ for every $n \in \mathbb{N}$, it follows that $\sup _{\lambda \in \Lambda} \phi\left(x_{0}, u_{n}(\lambda)\right) \leq \phi\left(x_{0}, x_{n}\right)+\xi_{n}$ for every $n \in \mathbb{N}$. Fix $\lambda \in \Lambda$ arbitrarily. From the assumption that $\lim _{\inf _{n \rightarrow \infty}} \alpha_{n}<1$, we may take subsequences $\left\{\alpha_{n_{i}}\right\}$ of $\left\{\alpha_{n}\right\}$ and $\left\{y_{n_{i}}(\lambda)\right\}$ of $\left\{y_{n}(\lambda)\right\}$ such that $\lim _{i \rightarrow \infty} \alpha_{n_{i}}=\alpha_{0}$ with $0 \leq \alpha_{0}<1$ and $\left\{J y_{n_{i}}(\lambda)\right\}$ converges weakly to a point $y_{0}^{*} \in E^{*}$. Then, by Lemma 3.1, we have that

$$
\lim _{i \rightarrow \infty}\left\|x_{n_{i}}-T_{\lambda}^{n_{i}} x_{n_{i}}\right\|=\lim _{i \rightarrow \infty}\left\|x_{0}-T_{\lambda}^{n_{i}} x_{n_{i}}\right\|=0 .
$$

From (3.23) and (3.24), we have

$$
\begin{aligned}
\left\|T_{\curlywedge}^{n_{i}+1} x_{n_{i}}-T_{\lambda}^{n_{i}} x_{n_{i}}\right\| \leq & \left\|T_{\lambda}^{n_{i}+1} x_{n_{i}}-T_{\lambda}^{n_{i}+1} x_{n_{i}+1}\right\|+\left\|T_{\curlywedge}^{n_{i}+1} x_{n_{i}+1}-x_{n_{i}+1}\right\| \\
& +\left\|x_{n_{i}+1}-x_{n_{i}}\right\|+\left\|x_{n_{i}}-T_{\lambda}^{n_{i}} x_{n_{i}}\right\| \\
\leq & \left(L_{\curlywedge}+1\right)\left\|x_{n_{i}+1}-x_{n_{i}}\right\|+\left\|T_{\curlywedge}^{n_{i}+1} x_{n_{i}+1}-x_{n_{i}+1}\right\|+\left\|x_{n_{i}}-T_{\lambda}^{n_{i}} x_{n_{i}}\right\| \\
& \longrightarrow 0 .
\end{aligned}
$$

Observe that

$$
\left\|T_{\lambda}^{n_{i}+1} x_{n_{i}}-x_{0}\right\| \leq\left\|T_{\lambda}^{n_{i}+1} x_{n_{i}}-T_{\lambda}^{n_{i}} x_{n_{i}}\right\|+\left\|T_{\lambda}^{n_{i}} x_{n_{i}}-x_{0}\right\| .
$$

By using (3.24),(3.25), and (3.26), we have

$$
\left\|T_{\lambda}^{n_{i}+1} x_{n_{i}}-x_{0}\right\| \longrightarrow 0, \quad \text { as } n \longrightarrow \infty
$$


Since $T_{\curlywedge}$ is uniformly Lipschitzian continuous, from (3.24) and (3.27), we have $x_{0}=T_{\lambda} x_{0}$, that is, $x_{0} \in \cap_{\lambda \in \Lambda} F\left(T_{\lambda}\right)$.

(b) Next we prove that $x_{0} \in \operatorname{EP}(G)$.

(1) In fact, since $x_{n} \rightarrow x_{0}$, we have

$$
\phi\left(x_{n+1}, x_{n}\right) \longrightarrow 0
$$

In view of $x_{n+1} \in C_{n+1}$, from the definition of $C_{n+1}$, we have

$$
\sup _{\lambda \in \Lambda} \phi\left(x_{n+1}, u_{n}(\lambda)\right) \leq \phi\left(x_{n+1}, x_{n}\right)+\xi_{n}
$$

From (3.28) and $\xi_{n} \rightarrow 0$, we have

$$
\sup _{\lambda \in \Lambda} \phi\left(x_{n+1}, u_{n}(\lambda)\right) \longrightarrow 0, \quad \forall \lambda \in \Lambda
$$

From (2.4), it yields $\sup _{\lambda \in \Lambda}\left(\left\|x_{n+1}\right\|-\left\|u_{n}(\lambda)\right\|\right)^{2} \rightarrow 0$. Since $\left\|x_{n+1}\right\| \rightarrow\left\|x_{0}\right\|$, we have

$$
\left\|u_{n}(\lambda)\right\| \longrightarrow\left\|x_{0}\right\|(n \longrightarrow \infty), \quad \forall \lambda \in \Lambda
$$

Hence we have

$$
\left\|J u_{n}(\lambda)\right\| \longrightarrow\left\|J x_{0}\right\|(n \longrightarrow \infty), \quad \forall \lambda \in \Lambda
$$

This implies that $\left\{J u_{n}(\lambda)\right\}$ is bounded in $E^{*}$. Since $E$ is reflexive, and so is $E^{*}$, we can assume that $J u_{n}(\lambda) \rightarrow f_{0} \in E^{*}$. In view of the reflexive of $E$, we see that $J(E)=E^{*}$. Hence there exists $p \in E$ such that $J p=f_{0}$. Since

$$
\begin{aligned}
\phi\left(x_{n+1}, u_{n}(\lambda)\right) & =\left\|x_{n+1}\right\|^{2}-2\left\langle x_{n+1}, J u_{n}(\lambda)\right\rangle+\left\|u_{n}(\lambda)\right\|^{2} \\
& =\left\|x_{n+1}\right\|^{2}-2\left\langle x_{n+1}, J u_{n}(\lambda)\right\rangle+\left\|J u_{n}(\lambda)\right\|^{2},
\end{aligned}
$$

taking $\liminf \operatorname{in}_{n \rightarrow \infty}$ on the both sides of equality above and in view of the weak lower semicontinuity of norm $\|\cdot\|$, it yields that

$$
\begin{aligned}
0 & \geq\left\|x_{0}\right\|^{2}-2\left\langle x_{0}, f_{0}\right\rangle+\left\|f_{0}\right\|^{2}=\left\|x_{0}\right\|^{2}-2\left\langle x_{0}, J p\right\rangle+\|J p\|^{2} \\
& =\left\|x_{0}\right\|^{2}-2\left\langle x_{0}, J p\right\rangle+\|p\|^{2}=\phi\left(x_{0}, p\right),
\end{aligned}
$$

that is, $x_{0}=p$. This implies that $f_{0}=J x_{0}$, and so $J u_{n}(\lambda) \rightarrow J x_{0}$, for all $\lambda \in \Lambda$. It follows from (3.32) and the Kadec-Klee property of $E^{*}$ that $J u_{n}(\lambda) \rightarrow J x_{0}(n \rightarrow \infty)$. Note that $J^{-1}: E^{*} \rightarrow$ $E$ is hemicontinuous, it yields that $u_{n}(\lambda) \rightarrow x_{0}$. It follows from (3.31) and the Kadec-Klee property of $E$ that

$$
\lim _{n \rightarrow \infty} u_{n}(\lambda)=x_{0}
$$


From (3.35), we have

$$
\lim _{n \rightarrow \infty}\left\|x_{n}-u_{n}(\lambda)\right\|=0, \quad \forall \lambda \in \Lambda
$$

Since $J$ is norm-to-norm continuous, hence we have that

$$
\left\|J x_{n}-J u_{n}(\lambda)\right\| \longrightarrow 0, \quad \forall \lambda \in \Lambda
$$

(2) Next we prove that

$$
\phi\left(u, x_{n}\right)-\phi\left(u, u_{n}(\lambda)\right) \longrightarrow 0, \quad \forall u \in F
$$

From (3.36) and (3.37), we have

$$
\begin{aligned}
\phi\left(u, x_{n}\right)-\phi\left(u, u_{n}(\lambda)\right) & =\left\|x_{n}\right\|^{2}-\left\|u_{n}(\lambda)\right\|^{2}-2\left\langle u, J x_{n}-J u_{n}(\lambda)\right\rangle \\
& \leq\left|\left\|x_{n}\right\|^{2}-\left\|u_{n}(\lambda)\right\|^{2}\right|+2\left|\left\langle u, J x_{n}-J u_{n}(\lambda)\right\rangle\right| \\
& \leq\left|\left\|x_{n}\right\|-\left\|u_{n}(\lambda)\right\|\right|\left(\left\|x_{n}\right\|+\left\|u_{n}(\lambda)\right\|\right)+2\|u\| \cdot\left\|J x_{n}-J u_{n}(\lambda)\right\| \\
& \leq\left\|x_{n}-u_{n}(\lambda)\right\|\left(\left\|x_{n}\right\|+\left\|u_{n}(\lambda)\right\|\right)+2\|u\| \cdot\left\|J x_{n}-J u_{n}(\lambda)\right\| . \\
& \longrightarrow 0 .
\end{aligned}
$$

Since

$$
\begin{aligned}
\phi\left(u_{n}(\lambda), y_{n}(\lambda)\right) & =\phi\left(W_{r_{n_{\lambda}}} y_{n}(\lambda), y_{n}(\lambda)\right) \\
& \leq \phi\left(u, y_{n}(\lambda)\right)-\phi\left(u, W_{r_{n_{\lambda}}} y_{n}(\lambda)\right) \\
& \leq \phi\left(u, x_{n}\right)+\xi_{n}-\phi\left(u, W_{r_{n_{\lambda}}} y_{n}(\lambda)\right) \\
& =\phi\left(u, x_{n}\right)+\xi_{n}-\phi\left(u, u_{n}(\lambda)\right),
\end{aligned}
$$

hence it follows from (3.38) and (3.40) that

$$
\lim _{n \rightarrow \infty} \phi\left(u_{n}(\lambda), y_{n}(\lambda)\right)=0 .
$$

From (2.3) and (3.41) it yields $\left(\left\|u_{n}(\lambda)\right\|-\left\|y_{n}(\lambda)\right\|\right)^{2} \rightarrow 0$. Since $\left\|u_{n}(\lambda)\right\| \rightarrow\left\|x_{0}\right\|$, we have

$$
\left\|y_{n}(\lambda)\right\| \longrightarrow\left\|x_{0}\right\|(n \longrightarrow \infty)
$$

Hence we have

$$
\left\|J y_{n}(\lambda)\right\| \longrightarrow\left\|J x_{0}\right\|(n \longrightarrow \infty)
$$


This implies that $\left\{J y_{n}(\lambda)\right\}$ is bounded in $E^{*}$. Since $E$ is reflexive, and so is $E^{*}$, we can assume that $J y_{n}(\lambda) \rightarrow g_{0} \in E^{*}$. In view of the reflexive of $E$, we see that $J(E)=E^{*}$. Hence there exists $y \in E$ such that $J y=g_{0}$. Since

$$
\begin{aligned}
\phi\left(u_{n}(\lambda), y_{n}(\lambda)\right) & =\left\|u_{n}(\lambda)\right\|^{2}-2\left\langle u_{n}(\lambda), J y_{n}(\lambda)\right\rangle+\left\|y_{n}(\lambda)\right\|^{2} \\
& =\left\|u_{n}(\lambda)\right\|^{2}-2\left\langle u_{n}(\lambda), J y_{n}(\lambda)\right\rangle+\left\|J y_{n}(\lambda)\right\|^{2}
\end{aligned}
$$

Taking $\lim \inf _{n \rightarrow \infty}$ on the both sides of equality above and in view of the weak lower semicontinuity of norm $\|\cdot\|$, it yields that

$$
\begin{aligned}
0 & \geq\left\|x_{0}\right\|^{2}-2\left\langle x_{0}, g_{0}\right\rangle+\left\|g_{0}\right\|^{2}=\left\|x_{0}\right\|^{2}-2\left\langle x_{0}, J y\right\rangle+\|J y\|^{2} \\
& =\left\|x_{0}\right\|^{2}-2\left\langle x_{0}, J y\right\rangle+\|y\|^{2}=\phi\left(x_{0}, y\right)
\end{aligned}
$$

that is, $x_{0}=y$. This implies that $g_{0}=J x_{0}$, and so $J y_{n}(\lambda) \rightarrow J x_{0}$. It follows from (3.43) and the Kadec-Klee property of $E^{*}$ that $J y_{n}(\lambda) \rightarrow J x_{0}(n \rightarrow \infty)$. Note that $J^{-1}: E^{*} \rightarrow E$ is hemicontinuous; it yields that $y_{n}(\lambda) \rightarrow x_{0}$. It follows from (3.42) and the Kadec-Klee property of $E$ that

$$
\lim _{n \rightarrow \infty} y_{n}(\lambda)=x_{0}
$$

Since $u_{n}(\lambda) \rightarrow x_{0}$, from (3.46), we have

$$
\lim _{n \rightarrow \infty}\left\|u_{n}(\lambda)-y_{n}(\lambda)\right\|=0
$$

Since $J$ is uniformly norm-to-norm continuous on bounded sets, from (3.47), we have

$$
\lim _{n \rightarrow \infty}\left\|J u_{n}(\lambda)-J y_{n}(\lambda)\right\|=0
$$

From $r_{n_{\curlywedge}} \geq a$, we have

$$
\lim _{n \rightarrow \infty} \frac{\left\|J u_{n}(\lambda)-J y_{n}(\lambda)\right\|}{r_{n_{\lambda}}}=0
$$

By $u_{n}(\lambda)=W_{r_{n}} y_{n}(\lambda)$, we have

$$
G\left(u_{n}(\lambda), y\right)+\frac{1}{r_{n_{\lambda}}}\left\langle y-u_{n}(\lambda), J u_{n}(\lambda)-J y_{n}(\lambda)\right\rangle \geq 0, \quad \forall y \in C
$$

From $\left(A_{2}\right)$, we have

$$
\frac{1}{r_{n_{\lambda}}}\left\langle y-u_{n}(\lambda), J u_{n}(\lambda)-J y_{n}(\lambda)\right\rangle \geq-G\left(u_{n}(\lambda), y\right) \geq G\left(y, u_{n}(\lambda)\right), \quad \forall y \in C
$$


Since $G(x, \cdot)$ is convex and lower semicontinuous, it is also weakly lower semicontinuous. So, letting $n \rightarrow \infty$, we have from (3.51) and $\left(\mathrm{A}_{4}\right)$ that

$$
G\left(y, x_{0}\right) \leq 0, \quad \forall y \in C
$$

For any $t$ with $0<t \leq 1$ and $y \in C$, let $y_{t}=t y+(1-t) x_{0}$. Since $y \in C$ and, hence, $G\left(y_{t}, x_{0}\right) \leq 0$, from conditions $\left(\mathrm{A}_{1}\right)$ and $\left(\mathrm{A}_{4}\right)$, we have

$$
0=G\left(y_{t}, y_{t}\right) \leq t G\left(y_{t}, y\right)+(1-t) G\left(y_{t}, x_{0}\right) \leq t G\left(y_{t}, y\right)
$$

This implies that $G\left(y_{t}, y\right) \geq 0$. Hence from condition $\left(\mathrm{A}_{3}\right)$, we have $G\left(x_{0}, y\right) \geq 0$ for all $y \in C$, and hence $x_{0} \in \operatorname{EP}(G)$.

Step 5. Finally we prove that $x_{n} \rightarrow P_{F} x$.

Since $x_{0}=P_{C_{0}} x \in F$ and $F$ is a nonempty closed convex subset of $C_{0}=\bigcap_{n=1}^{\infty} C_{n}$, we conclude that

$$
x_{0}=P_{F} x
$$

This completes the proof of Theorem 3.2.

The proof of Theorem 3.2 shows that the properties of projections used in the iterative scheme do not interact with the properties of mappings $\left\{T_{\lambda}\right\}$. Therefore, we may prove similar results as follows by replacing Theorem 2.4 with Theorem 2.5 in the proof.

Theorem 3.3. Let E be a strictly convex reflexive Banach space having the Kadec-Klee property and a Fréchet differentiable norm, $C$ a nonempty closed convex subset of $E, A: C \rightarrow E^{*}$ a continuous and monotone mapping, $\psi: C \rightarrow \mathbb{R}$ a lower semicontinuous and convex function, $F$ a bifunction from $C \times C$ to $\mathbb{R}$ which satisfies the conditions $\left(A_{1}\right)-\left(A_{4}\right)$ and let $\left\{T_{\lambda}\right\}(\lambda \in \Lambda): C \rightarrow C$ a family of uniformly Lipschitzian continuous and quasi- $\phi$-asymptotically nonexpansive mappings such that $F=\bigcap_{\lambda \in \Lambda} F\left(T_{\lambda}\right) \bigcap G M E P \neq \emptyset$. Assume that $R=\sup \{\|u\|: u \in F\}<\infty$. Let $\left\{x_{n}\right\}$ be the sequence generated by $x_{1}=x \in C, C_{1}=C$, and

$$
\begin{gathered}
y_{n}(\lambda)=J^{*}\left(\alpha_{n} J x_{n}+\left(1-\alpha_{n}\right) J T_{\lambda}^{n} x_{n}\right) \quad \forall \lambda \in \Lambda, \\
u_{n}(\lambda) \in C \text { such that } \\
F\left(u_{n}(\lambda), y\right)+\left\langle A u_{n}(\lambda), y-u_{n}(\lambda)\right\rangle+\psi(y)-\psi\left(u_{n}(\lambda)\right)+\frac{1}{r_{n_{\lambda}}}\left\langle y-u_{n}(\lambda), J u_{n}(\lambda)-J y_{n}(\lambda)\right\rangle \geq 0, \\
C_{n+1}=\{y \in C, \lambda \in \Lambda \\
\left.z \in C_{n}: \sup _{\lambda \in \Lambda} \phi\left(z, u_{n}(\lambda)\right) \leq \phi\left(z, x_{n}\right)+\xi_{n}\right\}, \\
x_{n+1}=\prod_{C_{n+1}} x, \quad \forall n \geq 0 .
\end{gathered}
$$


where $J$ is the duality mapping on $E$ and $\xi_{n}=\left(1-\alpha_{n}\right)\left(\sup _{\lambda \in \Lambda} k_{n}(\lambda)-1\right) \sup _{u \in F} \phi\left(u, x_{n}\right)$ for all $x_{n} \in$ $C$, where $\lim _{n \rightarrow \infty} \sup _{\lambda \in \Lambda} k_{n}(\lambda)=1$. Let $\left\{\alpha_{n}\right\}$ be a sequence in $[0,1]$ such that $\lim _{i n f} \inf _{n \rightarrow \infty} \alpha_{n}<1$ and $\left\{r_{n_{\lambda}}\right\} \subset[a, \infty)$ for some $a>0$, then $\left\{x_{n}\right\}$ converge strongly to $\Pi_{F} x$, where $\Pi_{F}$ is the generalized projection of $E$ onto $F$.

\section{Acknowledgments}

The authors would like to express their thanks to the referees for their helpful suggestions and comments.

\section{References}

[1] L.-C. Ceng and J.-C. Yao, "A hybrid iterative scheme for mixed equilibrium problems and fixed point problems," Journal of Computational and Applied Mathematics, vol. 214, no. 1, pp. 186-201, 2008.

[2] S. Takahashi and W. Takahashi, "Strong convergence theorem for a generalized equilibrium problem and a nonexpansive mapping in a Hilbert space," Nonlinear Analysis: Theory, Methods E Applications, vol. 69, no. 3, pp. 1025-1033, 2008.

[3] A. Tada and W. Takahashi, "Weak and strong convergence theorems for a nonexpansive mapping and an equilibrium problem," Journal of Optimization Theory and Applications, vol. 133, no. 3, pp. 359-370, 2007.

[4] S. Takahashi and W. Takahashi, "Viscosity approximation methods for equilibrium problems and fixed point problems in Hilbert spaces," Journal of Mathematical Analysis and Applications, vol. 331, no. 1, pp. 506-515, 2007.

[5] W. Takahashi and K. Zembayashi, "Strong convergence theorem by a new hybrid method for equilibrium problems and relatively nonexpansive mappings," Fixed Point Theory and Applications, vol. 2008, Article ID 528476, 11 pages, 2008.

[6] Y. Kimura and W. Takahashi, "On a hybrid method for a family of relatively nonexpansive mappings in a Banach space," Journal of Mathematical Analysis and Applications, vol. 357, no. 2, pp. 356-363, 2009.

[7] W. Takahashi, Nonlinear Functional Analysis, Fixed Point Theory and Its Applications, Yokohama Publishers, Yokohama, Japan, 2000.

[8] K. Goebel and S. Reich, Uniform Convexity, Hyperbolic Geometry, and Nonexpansive Mappings, vol. 83 of Monographs and Textbooks in Pure and Applied Mathematics, Marcel Dekker, New York, NY, USA, 1984.

[9] U. Mosco, "Convergence of convex sets and of solutions of variational inequalities," Advances in Mathematics, vol. 3, pp. 510-585, 1969.

[10] G. Beer, Topologies on Closed and Closed Convex Sets, vol. 268 of Mathematics and Its Applications, Kluwer Academic Publishers, Dordrecht, The Netherlands, 1993.

[11] Y. I. Alber, "Metric and generalized projection operators in Banach spaces: properties and applications," in Theory and Applications of Nonlinear Operators of Accretive and Monotone Type, A. G. Kartsators, Ed., vol. 178 of Lecture Notes in Pure and Applied Mathematics, pp. 15-50, Marcel Dekker, New York, NY, USA, 1996.

[12] S. Kamimura and W. Takahashi, "Strong convergence of a proximal-type algorithm in a Banach space," SIAM Journal on Optimization, vol. 13, no. 3, pp. 938-945, 2002.

[13] M. Tsukada, "Convergence of best approximations in a smooth Banach space," Journal of Approximation Theory, vol. 40, no. 4, pp. 301-309, 1984.

[14] Y. Su and X. Qin, "Strong convergence of modified Ishikawa iterations for nonlinear mappings," Proceedings of the Indian Academy of Sciences. Mathematical Sciences, vol. 117, no. 1, pp. 97-107, 2007.

[15] Y. Censor and S. Reich, "Iterations of paracontractions and firmly nonexpansive operators with applications to feasibility and optimization," Optimization, vol. 37, no. 4, pp. 323-339, 1996.

[16] S. Reich, "A weak convergence theorem for the alternating method with Bregman distances," in Theory and Applications of Nonlinear Operators of Accretive and Monotone Type, A. G. Kartsators, Ed., vol. 178 of Lecture Notes in Pure and Applied Mathematics, pp. 313-318, Marcel Dekker, New York, NY, USA, 1996. 
[17] S. Matsushita and W. Takahashi, "Weak and strong convergence theorems for relatively nonexpansive mappings in Banach spaces," Fixed Point Theory and Applications, vol. 2004, no. 1, pp. 37-47, 2004.

[18] E. Blum and W. Oettli, "From optimization and variational inequalities to equilibrium problems," The Mathematics Student, vol. 63, no. 1-4, pp. 123-145, 1994. 